\title{
Errata: Fluctuations in Nonequilibrium Systems and Broken Supersymmetry ${ }^{1}$
}

M. F. Zimmer ${ }^{2}$

Received February 24, 1994

A number of typographical errors appeared in this paper. They are clarified below.

1. Equation (33) should read

$$
\frac{1}{\varepsilon^{\prime}}\langle\delta S\rangle_{\mathcal{s}}=\int d x\left\langle J_{\alpha} L_{\beta \alpha} \bar{C}_{\beta}+\tilde{J}_{\alpha} \dot{\bar{C}}_{\alpha}+K_{\alpha}\left(L_{\beta \alpha} \tilde{\psi}_{\beta}-\dot{\psi}_{\alpha}\right)\right\rangle_{\mathcal{S}}
$$

2. The $G$ in Eq. (34) should actually be $F$.

3. The $\tilde{\psi}$ in the third term on the left-hand side of Eq. (36) should be $\widetilde{\psi}_{\beta}$.

4. Equation 5 should read

$$
\partial, \psi=-\Gamma_{0} \frac{\delta A}{\delta \psi}-F[M]+v
$$

5. Following Eq. (17), the expression in the text for $C_{0}\left(t_{1}, t_{2}\right)$ should be $\left(\Gamma_{0} / \omega\right)\left[G_{0}\left(t_{1}, t_{2}\right)+G_{0}\left(t_{2}, t_{1}\right)\right]$.

6. In (43) the variation of $C$ should be $\delta C=-\varepsilon^{\prime}(\dot{\psi}+D \Delta \tilde{\psi})$.

In addition, the author wishes to thank a referee for making a helpful observation with regard to the original version of Eq. (16).

\footnotetext{
' This paper originally appeared in J. Stat. Phys. 73:751 (1993).

${ }^{2}$ IFF, Forschungszentrum Jülich, D-52425 Jülich, Germany. e-mail: IFF121@zam001.zam. kfa-juelich.de.
} 